\title{
Discharge delay in patients with community-acquired pneumonia managed on a critical pathway
}

\author{
Jeremy J Moeller MD¹, Martin Ma MD ${ }^{1}$, Paul Hernandez MDCM FRCPC ${ }^{2}$, Thomas Marrie MD FRCPC ${ }^{3}$, \\ Claire Touchie MD FRCPC ${ }^{4}$, Ward Patrick MD FRCPC ${ }^{2}$
}

\begin{abstract}
JJ Moeller, M Ma, P Hernandez, T Marrie, C Touchie, W Patrick. Discharge delay in patients with community-acquired pneumonia managed on a critical pathway. Can J Infect Dis Med Microbiol 2006;17(2):109-113.
\end{abstract}

INTRODUCTION: It has previously been reported that a critical pathway for community-acquired pneumonia (CAP) significantly reduces bed days per patient managed but results in no difference in average length of stay, suggesting that discharge criteria were not successfully implemented. The present study sought to identify factors in the timing of discharge not taken into account by discharge criteria.

METHODS: Patients admitted with CAP and placed on a pneumonia critical pathway were studied. Patients' functional and cognitive status were evaluated using the Barthel Index, Hierarchical Assessment of Balance and Mobility (HABAM) and the Mini-Mental Status Examination. Once discharge criteria were met, the patient, a family member and the treating physician were interviewed to identify other factors contributing to length of stay.

RESULTS: Thirty-one patients were enrolled in the study; 12 were discharged when they met discharge criteria and 19 stayed in hospital longer. There were no differences between patients discharged at stability versus those with an increased length of stay in terms of demographics, pneumonia severity score, functional or cognitive status at discharge using the Barthel Index $(87.3 \pm 11.1$ versus $83.8 \pm 8.6$, respectively; $\mathrm{P}=0.46)$ and MMSE (27.1 \pm 1.1 versus $27.3 \pm 1.1$, respectively; $\mathrm{P}=0.64)$; however, there was a significant difference in HABAM score at the time clinical stability was reached $(22.6 \pm 1.3$ versus $17.4 \pm 3.5$, respectively; $\mathrm{P}=0.03)$, which correlated with physician and family assessments of patients' readiness for discharge.

CONCLUSIONS: HABAM may be a useful tool to identify patients at risk of remaining in hospital after objective discharge criteria are met. Additional resources may be targeted at these patients to reduce length of stay in CAP.

Key Words: Community-acquired pneumonia; Critical pathway; Discharge criteria; Length of stay

\section{Un délai avant d'obtenir le congé chez des patients atteints de pneumonie non nosocomiale pris en charge par chemin critique}

INTRODUCTION : Il a déjà été déclaré qu'un chemin critique en cas de
pneumonie non nosocomiale (PNN) réduit considérablement la durée
d'hospitalisation par patient pris en charge mais ne change en rien le séjour
hospitalier moyen, laissant supposer que les critères d'attribution du congé
n'étaient pas respectés. La présente étude visait à repérer les facteurs
relatifs au moment du congé qui n'étaient pas pris en compte dans les
critères d'attribution du congé. MÉTHODOLOGIE : Les patients hospitalisés en raison d'une PNN et respectant un chemin critique ont été étudiés. Leur état fonctionnel et cognitif a été évalué selon l'indice de Barthel, l'évaluation hiérarchique de l'équilibre et de la mobilité (ÉHÉM) et le mini-examen de l'état mental (MEÉM). Lorsque les critères d'attribution du congé étaient respectés, le patient, un membre de la famille et le médecin traitant étaient interviewés pour repérer d'autres facteurs contribuant à la durée d'hospitalisation.

RÉSULTATS : Trente et un patients ont participé à l'étude. Douze ont quitté l'hôpital lorsqu'ils respectaient les critères d'attribution du congé et 19 sont demeurés hospitalisés plus longtemps. Il n'existait aucune différence entre les patients obtenant leur congé à l'atteinte de la stabilité et ceux demeurant à l'hôpital plus longtemps pour ce qui est de la démographie, de l'indice de gravité de la pneumonie, de l'état fonctionnel ou cognitif au congé d'après l'indice de Barthel $(87,3 \pm 11,1$ par rapport à $83,8 \pm 8,6$, respectivement; $\mathrm{P}=0,46)$ et le MEÉM $(27,1 \pm 1,1$ par rapport à $27,3 \pm 1,1$, respectivement; $\mathrm{P}=0,64)$. On remarquait toutefois une différence considérable dans l'indice d'ÉHÉM au moment de l'atteinte de la stabilité clinique $(22,6 \pm 1,3$ par rapport à $17,4 \pm 3,5$, respectivement; $\mathrm{P}=0,03)$, ce qui était corrélé par les évaluations du médecin et de la famille de l'état de préparation du patient au congé.

CONCLUSION : L'ÉHÉM pourrait contribuer à repérer les patients qui risquent de demeurer hospitalisés après l'atteinte des critères objectifs d'attribution du congé. Des ressources supplémentaires pourraient être ciblées chez ces patients atteints de PNN afin de réduire leur durée d'hospitalisation.

annually (1). According to various economic analyses, over $90 \%$ of the cost is due to the provision of care for those hospitalized with CAP (2-4).

Although the concept of pneumonia as a clinical entity has been understood for many years, the diagnosis and management of patients with pneumonia remains controversial $(5,6)$. Many studies have shown that there is significant variation in the way patients with CAP are treated in-hospital. In particular, there are significant differences in the median length of stay (LOS) among hospitals (7-11). In a retrospective study of CAP in Canada has been estimated to be $\$ 100$ million

${ }^{1}$ Faculty of Medicine; ${ }^{2}$ Department of Medicine, Dalhousie University, Halifax, Nova Scotia; ${ }^{3}$ Department of Medicine, University of Alberta, Edmonton, Alberta; ${ }^{4}$ Department of Medicine, University of Ottawa, Ottawa, Ontario

Correspondence: Dr Ward Patrick, Room 377, Bethune Building, Victoria General Hospital Site, 1278 Tower Road, Queen Elizabeth II Health

Sciences Centre, Halifax, Nova Scotia B3H 2Y9. Telephone 902-473-3606, fax 902-473-3610, e-mail ward.patrick@cdha.nshealth.ca

Received for publication July 12, 2005. Accepted February 27, 2006 
patients with CAP in 20 Canadian hospitals, Feagan et al (12) showed that median LOS ranged from 5.0 to 9.0 days. LOS has been reported to depend on a number of factors independent of the patient, such as geographic location (eg, treatment in an urban or rural hospital) (10), the treating physician's specialty (eg, primary care physician or hospitalist) (11) and the opinion of the attending physician $(8,9)$.

To increase the efficiency and uniformity of CAP treatment, efforts have been made to develop a critical pathway for the management of patients with CAP. The three major components of a CAP critical pathway, developed by Marrie et al (14), are the Pneumonia Severity Index admission rule of Fine et al (13), treatment with broad-spectrum, empirical antibiotic therapy (such as with a respiratory fluoroquinolone) and practice guidelines for daily inpatient care. The practice guidelines consist of criteria for switching from intravenous to oral antibiotic therapy and for discharge from hospital. In a study in which 19 Canadian hospitals were randomly assigned to either conventional pneumonia treatment or a critical pathway (14), there were no significant differences in terms of outcomes of intensive care unit admission, mortality, readmissions or complications of the disease. Hospitals that used the critical pathway had an 18\% absolute reduction in the admission of low-risk patients versus hospitals that used conventional treatment (31\% versus $48 \%$, respectively), while the rate of admission of high-risk patients was similar between the two groups. There was also a significant reduction in the number of bed days per patient managed in hospitals using the critical pathway versus those using conventional treatment (4.4 versus 6.1 days, respectively; $\mathrm{P}=0.04)$. In hospitals in which the critical pathway was used, up to one-half of all inpatients were not discharged upon meeting stabilization criteria. Economic analyses performed in the United States have shown that a one-day reduction in LOS may yield substantial cost savings for both the hospital and the patient (15) without necessarily compromising patient outcomes $(16,17)$.

The present study was designed to assess patient- and physician-related barriers to the discharge of patients who have met the appropriate objective criteria. By identifying the barriers to the optimal usage of the critical pathway for CAP, we can begin to implement strategies to improve treatment in a cost-effective manner.

\section{Study site and patients}

\section{METHODS}

The study was conducted from July 1999 to August 2001 at the Queen Elizabeth II Health Sciences Centre, a 637-bed, university-affiliated hospital in Halifax, Nova Scotia. The institution's research ethics board approved the study protocol. Patients were eligible for inclusion in the study if they met the following criteria: admission through the emergency department with a primary diagnosis of CAP; treatment on the institution's pneumonia critical pathway; and the ability to respond to various questionnaires and give informed consent. CAP was defined as symptoms and signs consistent with a lower respiratory tract infection associated with a new pulmonary opacity on chest radiograph compatible with pneumonia, as interpreted by a radiologist at the Queen Elizabeth II Health Sciences Centre. Exclusion criteria were an immunocompromised state (ie, HIV infection, use of over $10 \mathrm{mg} /$ day of prednisone or other immunosuppressive agents, history of organ transplantation, active treatment for cancer or alcohol addiction), active tuberculosis, cystic fibrosis, nosocomial pneumonia, requirement of direct intensive care unit admission, chronic renal failure (estimated creatinine clearance less than $20 \mathrm{~mL} / \mathrm{min}$ ), pregnancy or lactation, and inability to communicate adequately to complete the study protocol or give informed consent.

\section{Functional and cognitive evaluation}

Three tests were used to evaluate the functional and cognitive capacity of each study patient during their hospital stay: the Barthel Index (BI) $(18,19)$, the Hierarchical Assessment of Balance and Mobility (HABAM) (20) and the Mini-Mental State Examination (MMSE) (21).

The BI includes an evaluation of 15 self care, sphincter control and mobility factors. The patient is asked whether he or she is capable of each of the 15 functions, and the scoring is subdivided based on a response of 'can do by myself', 'can do with the help of someone else' and 'cannot do at all'. The total BI score ranges from zero (total dependence) to 100 (complete independence) $(18,19)$.

The HABAM was developed to graphically display changes in a patient's balance and mobility. Patients are asked about their maximum capabilities in terms of balance, transfers and mobility. For the purpose of statistical analysis, numerical values are assigned to the patient's level of balance, mobility and transfer capability. The maximum total score is 24 , indicating normal balance and mobility.

Patients' cognitive function was assessed using the MMSE. The MMSE is scored out of a maximum of 30 points; a score of 24 to 30 indicates the normal range, and scores of 20 to 23,11 to 19 and zero to 10 represents mild, moderate and severe cognitive impairment, respectively.

\section{Discharge criteria}

Clinical stability and suitability for discharge were determined based on the criteria developed by Marrie et al (14). By these criteria, a patient is considered ready for discharge when his vital signs have normalized, lung function and oxygenation have returned to baseline status, blood culture is negative, blood cell count is lower than $12 \times 10^{9} / \mathrm{L}$ and any comorbid illnesses have stabilized (Table 1).

\section{Assessment of patients}

Patients meeting the eligibility criteria for the study were identified, informed consent was obtained and interviews were conducted within $48 \mathrm{~h}$ of admission to hospital. Demographic, clinical and laboratory data were obtained directly from patient interviews and from hospital chart review. Finally, within 48 h of admission to hospital, the BI, HABAM and MMSE were used to provide a retrospective assessment of premorbid functional and cognitive status.

These latter three assessments were performed again once the patient reached clinical stability according to the CAP critical pathway criteria. The patient was also interviewed using a semistructured question format when clinical stability was met. Patients were asked whether they felt ready to go home and whether they had any help at home. They were also asked to describe any fears or concerns they had about going home, if they had any. Finally, patients rated the quality of care they received in-hospital, and were asked whether they would have preferred to receive all of their treatment at home. 
TABLE 1

Discharge criteria for patients hospitalized with community-acquired pneumonia

Able to eat and drink
Temperature $\leq 38^{\circ} \mathrm{C}$ for the previous $16 \mathrm{~h}$
Respiratory rate $\leq 24 / \mathrm{min}$ for the previous $16 \mathrm{~h}$
Pulse rate $\leq 100 / \mathrm{min}$ for the previous $16 \mathrm{~h}$
Negative blood culture
Oxygenation normal (or baseline for subject), such that oxygen saturation
$>90 \%$ while breathing room air. For subjects with COPD, the $\mathrm{pO}_{2}$ should
be $>60 \mathrm{mmHg}$ and $\mathrm{pCO}_{2}<45 \mathrm{mmHg}$ or there should be a return to
previous baseline
White blood cell count $<12 \times 10^{9} / \mathrm{L}$
Stable comorbid illness

COPD Chronic obstructive pulmonary disease; $\mathrm{pCO}_{2}$ Partial pressure of carbon dioxide; $\mathrm{pO}_{2}$ Partial pressure of oxygen

\section{TABLE 2}

Baseline characteristics of subjects hospitalized with community-acquired pneumonia

\begin{tabular}{lccc}
\hline & $\begin{array}{c}\text { Patients discharged } \\
\text { at time of } \\
\text { stability (n=12) } \\
\text { (n [\%]) }\end{array}$ & $\begin{array}{c}\text { Patients with } \\
\text { increased } \\
\text { length of stay (n=19) } \\
\text { (n [\%]) }\end{array}$ & P \\
\hline Age $\geq 65$ years & $9(75)$ & $13(68)$ & 0.69 \\
Male sex & $4(33)$ & $10(53)$ & 0.29 \\
Severity of illness on admission & & & \\
Risk class I & $1(8)$ & $1(5)$ & \\
Risk class II & $1(8)$ & $2(11)$ & \\
Risk class III & $1(8)$ & $6(32)$ & \\
Risk class IV & $6(50)$ & $8(42)$ & \\
Risk class V & $3(25)$ & $2(11)$ & 0.56 \\
\hline
\end{tabular}

A family member was also contacted and interviewed when the patient reached clinical stability. The family member was asked to determine whether they felt the patient was ready to go home and to describe the impact that the patient's discharge would have on them.

If the patient was not discharged within $16 \mathrm{~h}$ of reaching clinical stability, then the treating physician was interviewed. Physicians were asked to give reasons for the extension of the patient's stay, about their knowledge of the pneumonia critical pathway and about their level of experience in treating CAP.

If the patient stayed in hospital after reaching clinical stability, then the functional assessment was performed once again before the patient was finally discharged.

\section{Statistical analysis}

Several statistical methods were used to evaluate the results. Pearson's $\chi^{2}$ analysis was used to compare proportions, while Student's $t$ test was used to compare means between the two groups. $\mathrm{P}<0.05$ was considered statistically significant.

\section{RESULTS}

There were three recruitment periods for the study: between July 1 and August 13, 1999; between May 31 and August 17, 2000; and between July 1 and August 10, 2001. During these periods, 142 potential cases of CAP were identified. Forty-five
TABLE 3

Functional and cognitive status, and length of stay data

\begin{tabular}{|c|c|c|c|}
\hline & $\begin{array}{c}\text { Patients } \\
\text { discharged } \\
\text { at time of } \\
\text { stability }(n=12)\end{array}$ & $\begin{array}{l}\text { Patients with } \\
\text { increased } \\
\text { length } \\
\text { of stay }(n=19)\end{array}$ & $\mathbf{P}$ \\
\hline \multicolumn{4}{|c|}{ Mean Barthel Index score (maximum 100) } \\
\hline Before admission & 88.9 & 87.8 & 0.95 \\
\hline Within $48 \mathrm{~h}$ of admission & 69.6 & 73.6 & 0.46 \\
\hline At stability & $87.3(n=10)$ & $83.8(n=17)$ & 0.46 \\
\hline Upon discharge from hospital & $86.9^{*}$ & 87.4 & 0.92 \\
\hline \multicolumn{4}{|l|}{ Mean MMSE score (maximum 30) } \\
\hline Within $48 \mathrm{~h}$ of admission & 27.5 & 26.9 & 0.59 \\
\hline At stability & $27.1(n=10)$ & $27.3(n=17)$ & 0.64 \\
\hline Upon discharge from hospital & $27.2^{*}$ & 27.7 & 0.29 \\
\hline \multicolumn{4}{|c|}{ Mean HABAM score (maximum 24) ${ }^{\dagger}$} \\
\hline Before admission & 22.7 & 19.8 & 0.15 \\
\hline Within $48 \mathrm{~h}$ of admission & 20.1 & 15.9 & 0.07 \\
\hline At stability & 22.6 & 17.4 & 0.03 \\
\hline Upon discharge from hospital & $22.5^{\star}$ & 19.7 & 0.07 \\
\hline Mean length of stay, days & 6.7 & 7.9 & \\
\hline Median length of stay, days & 5.5 & 7.5 & \\
\hline Mean time to stability, days & 6.3 & 4.2 & \\
\hline Median time to stability, days & 5.0 & 4.0 & \\
\hline
\end{tabular}

${ }^{*}$ Patients discharged from hospital within $16 \mathrm{~h}$ of reaching clinical stability were considered discharged at time of stability; ${ }^{\dagger}$ The Hierarchical Assessment of Balance and Mobility (HABAM) score was obtained from only 18 of 19 patients with increased length of stay. MMSE Mini-Mental Status Examination

patients $(31.7 \%)$ were enrolled, but 14 patients were subsequently excluded from the study. Of the patients excluded from the study, two died in hospital, two withdrew, two were transferred to other hospital units, two patients were reviewed and considered unlikely to have pneumonia, and six patients were lost to follow-up. Of the 97 patients not enrolled in the study, the most common reasons for exclusion were inability to communicate $(n=50)$ and refusal to give informed consent $(n=38)$.

Of the patients who were included in the study, 12 (38.7\%) were discharged from hospital upon reaching stability, and $19(61.3 \%)$ stayed in hospital after reaching stability. Baseline demographic characteristics were similar between groups (Table 2). Additionally, there were no differences in highest education level obtained, employment status, marital status and premorbid living arrangements. There were no significant differences in rates of respiratory and other comorbid conditions. Both groups had a similar profile for the Pneumonia Severity Index at time of admission (Table 2), although $75 \%$ of patients discharged upon reaching clinical stability were admitted in risk classes IV and V, compared with $53 \%$ of patients with increased LOS $(\mathrm{P}>0.05)$.

Initial clinical assessment, including symptoms, signs and laboratory values (complete blood cell counts, biochemistry and arterial blood gas values) upon presentation to hospital did not help to distinguish between patients discharged at time of stability and those with increased LOS.

Table 3 shows that there were no significant differences in baseline scores of the BI or the MMSE between groups. Some of the study patients were unable to perform the MMSE due to either visual impairment or illiteracy. In addition, both groups 
reached baseline values for the BI and MMSE by the time they reached clinical stability. The mean HABAM score at clinical stability was significantly lower in the group of patients with an increased LOS (17.4 versus 22.6, $\mathrm{P}<0.05)$. In addition, in the increased LOS group, the HABAM score increased by an average of 2.3 points between the time patients reached clinical stability and the time they were eventually discharged from hospital. Mean and median values for LOS in both groups are also presented in Table 3.

Patient interviews revealed that $58 \%$ of the patients with a prolonged LOS felt that they were ready to go home upon reaching clinical stability, compared with $92 \%$ of patients without discharge delay $(\mathrm{P}<0.05)$. No specific concern about patients' ability to perform basic activities of daily living accounted for this difference. Some patients' families could not be contacted (one of 12 families of patients discharged at stability versus four of 19 families of patients with prolonged LOS). Only $40 \%$ of the family members of patients with a prolonged LOS felt that their family member was ready for discharge, compared with $82 \%$ of family members of patients discharged without delay $(\mathrm{P}<0.05)$.

Twelve of the 19 treating physicians of patients who were not discharged upon reaching clinical stability were interviewed. Four of these physicians felt that their patient was ready to go home but were kept in-hospital for various reasons: one discharge was delayed because additional pulmonary function tests were required; two patients reported feeling unready for discharge to their physician; and one patient had a delay in establishing home supports. Eight physicians did not think that their patient was ready to go home: three patients were considered too frail; three patients were kept to monitor comorbid conditions; one patient had intractable nausea; and there were concerns that one patient would not comply with treatment at home. All of the physicians interviewed were postgraduate trainees who had graduated from medical school within the previous five years. Just three of the 12 physicians interviewed were aware of the discharge guidelines for CAP in use at the institution.

\section{DISCUSSION}

According to the study by Marrie et al (14), approximately one-half of all pneumonia inpatients are not discharged upon reaching clinical stability. Our data confirms and further extends this finding.

Four conclusions can be made from our data. First, there seems to be little difference in the objective clinical or demographic characteristics between patients with an increased LOS and those who were discharged upon reaching clinical stability. The patients who were discharged earlier were no less acutely ill; specifically, $75 \%$ of patients discharged at the time of clinical stability were in risk classes IV and V, compared with $53 \%$ of those patients with an increased LOS.

Second, the functional and cognitive status of patients, as measured by the BI and MMSE, does not improve significantly when patients are kept in-hospital after they have reached clinical stability. However, our results suggest that patients with a prolonged LOS have a lower HABAM score at the time of admission to hospital. In addition, this score did not return to prehospitalization values by the time the patient reached clinical stability. This suggests that the HABAM may be an important predictor for prolonged LOS in patients with CAP.
This result also suggests a potential role for physiotherapy and other support services to allow patients to reach optimal functional status while in-hospital.

Third, while patients with an increased LOS stayed an average of one day longer than patients without prolonged LOS, patients with increased LOS reached clinical stability one to two days earlier. This is consistent with the result that fewer patients in the increased LOS group had pneumonia risk classification scores in classes IV and V. Patients with increased LOS may have had less severe pneumonia, and their prolonged hospital LOS may have been due to other factors, such as perception of poor balance and mobility.

Fourth, the opinions of patients' family members seem to correlate with the decision to discharge the patient. Several suggestions may explain this finding. It is likely that family members exerted a significant influence on physician practices, including the decision to discharge a patient. Physicians may have believed that family members were a better judge of the ability of patients to cope at home than the hospital staff or the patient. Physicians may wish to temporarily reduce the burden of caregiving from family members. If this is the case, then additional home support services may be of benefit to families and may be potentially less expensive than providing hospital-based care. It is necessary to explore further the relationship between the impressions of family members and physician decision-making in the setting of CAP.

There are several limitations to the present study. The relatively small sample size limits the power of the study to clearly identify all of the factors that explain discharge delay. For some assessments (eg, MMSE, physician and family interviews), missing data further reduced results available for review. Additionally, the study was dependent on the patient's ability to communicate and respond to detailed questionnaires. Therefore, our findings are limited to patients without dementia, severe mental handicaps or other difficulties with communication. Our study population is therefore not representative of all patients hospitalized with CAP. Finally, we used the BI and HABAM, which are self-reported measures of aspects of functional capacity. It is possible that the patients over- or underestimated their ability to perform each activity.

CAP is a common and costly reason for hospitalization. Standardization of care through implementation of a critical pathway can improve patient outcomes and reduce the use of health care resources. Discharge delays, however, remain an important problem. Targeting patients with poor functional capacity with additional inpatient (eg, physiotherapy) and outpatient (eg, home support) services may facilitate earlier discharge once clinical stability has been reached. Using a simple clinical tool such as the HABAM may help to identify these patients within the first two days of admission to hospital. Further, multicentre studies with larger sample sizes are needed to confirm the findings of the present study. As well, studies evaluating interventions (eg, inpatient physiotherapy, additional home supports) directed toward patients at increased risk for discharge delay are required.

ACKNOWLEDGEMENTS: Jeremy J Moeller and Martin Ma are recipients of the Dalhousie University summer student research grant. 


\section{REFERENCES}

1. Health Canada. Respiratory Diseases in Canada. Ottawa: Health Canada, 2001.

2. Lave JR, Lin CJ, Hughes-Cromick P, Fine MJ, et al. The cost of treating patients with community-acquired pneumonia. Semin Respir Crit Care Med 1999;20:189-97.

3. Niederman MS, McCombs JS, Unger AN, Kumar A, Popovian R. The cost of treating community-acquired pneumonia. Clin Ther 1998;20:820-37.

4. Bartolome M, Almirall J, Morera J, et al; Maresme CommunityAcquired Pneumonia Study Group (GEMPAC). A population-based study of the costs of care for community-acquired pneumonia. Eur Respir J 2004:23:610-6.

5. Ost D, Farber BF, Fein A, Grossman R, Cassiere H, Fein A. Diagnosis and management of pneumonia and other respiratory infections, 1st edn. Oklahoma: Professional Communications, 1999.

6. LaForce FM. Triaging care for patients with pneumonia. Real progress. Arch Intern Med 1997;157:15-6.

7. Rhew DC, Tu GS, Ofman J, Henning JM, Richards MS, Weingarten SR. Early switch and early discharge strategies in patients with community-acquired pneumonia: A meta-analysis. Arch Intern Med 2001;161:722-7.

8. Laing R, Coles C, Chambers S et al. Community-acquired pneumonia: Influence of management practices on length of hospital stay. Intern Med J 2004;34:91-7.

9. Cabre M, Bolivar I, Pera G, Pallares R; Pneumonia Study Collaborative Group. Factors influencing length of hospital stay in community-acquired pneumonia: A study in 27 community hospitals. Epidemiol Infect 2004;132:821-9.

10. Jin Y, Marrie TJ, Carriere KC, et al. Variation in management of community-acquired pneumonia requiring admission to Alberta, Canada hospitals. Epidemiol Infect 2003;130:41-51.

11. Rifkin WD, Conner D, Silver A, Eichorn A. Comparison of processes and outcomes of pneumonia care between hospitalists and community-based primary care physicians. Mayo Clin Proc 2002;77:1053-8.

12. Feagan BG, Marrie TJ, Lau CY, Wheeler SL, Wong CJ, Vandervoort MK. Treatment and outcomes of communityacquired pneumonia at Canadian hospitals. CMAJ 2000;162:1415-20.

13. Fine MJ, Auble TE, Yealy DM, et al. A prediction rule to identify low-risk patients with community-acquired pneumonia. N Engl J Med 1997;336:243-50.

14. Marrie TJ, Lau CY, Wheeler SL, Wong CJ, Vandervoort MK, Feagan BG. A controlled trial of a critical pathway for treatment of community-acquired pneumonia. CAPITAL Study Investigators. JAMA 2000;283:749-55.

15. Fine MJ, Pratt HM, Obrosky DS, et al. Relation between length of hospital stay and costs of care for patients with community-acquired pneumonia. Am J Med 2000;109:378-85.

16. Marras TK, Gutierrez C, Chan CK. Applying a prediction rule to identify low-risk patients with community-acquired pneumonia. Chest 2000;118:1339-43.

17. McCormick D, Fine MJ, Coley CM, et al. Variation in length of hospital stay in patients with community-acquired pneumonia: Are shorter stays associated with worse medical outcomes? Am J Med 1999;107:5-12.

18. Mahoney FI, Barthel DW. Functional evaluation: The Barthel Index. Md State Med J 1965;14:61-5.

19. Granger CV, Albrecht GL, Hamilton BB. Outcome of comprehensive medical rehabilitation: Measurement by PULSES profile and the Barthel Index. Arch Phys Med Rehabil 1979;60:145-54.

20. MacKnight C, Rockwood K. A hierarchical assessment of balance and mobility. Age Ageing 1995;24:126-30.

21. Folstein MF, Folstein SE, McHugh PR. "Mini-mental state". A practical method for grading the cognitive state of patients for the clinician. J Psychiatr Res 1975;12:189-98. 


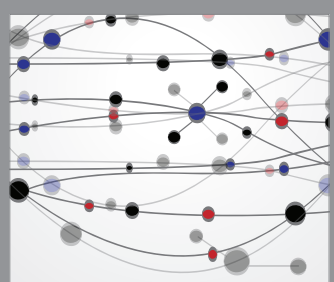

The Scientific World Journal
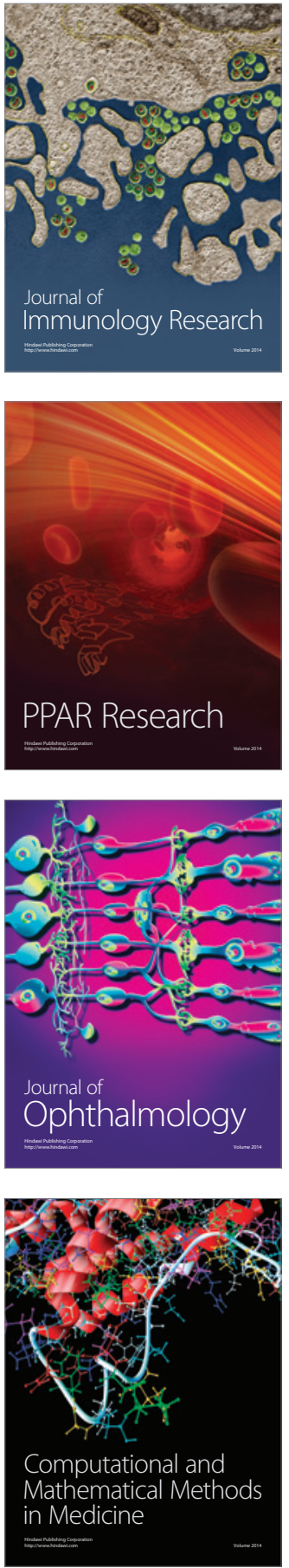

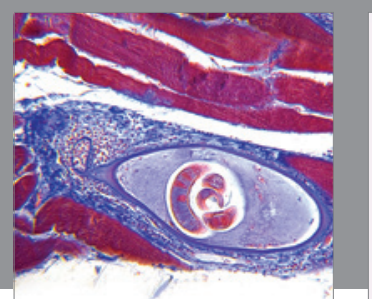

Gastroenterology Research and Practice

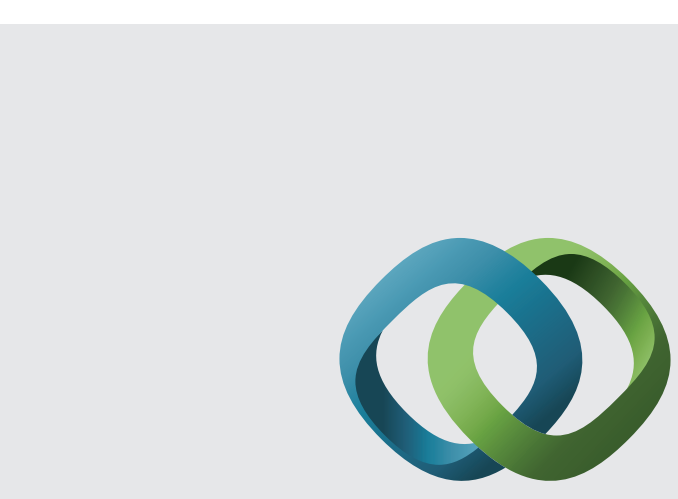

\section{Hindawi}

Submit your manuscripts at

http://www.hindawi.com
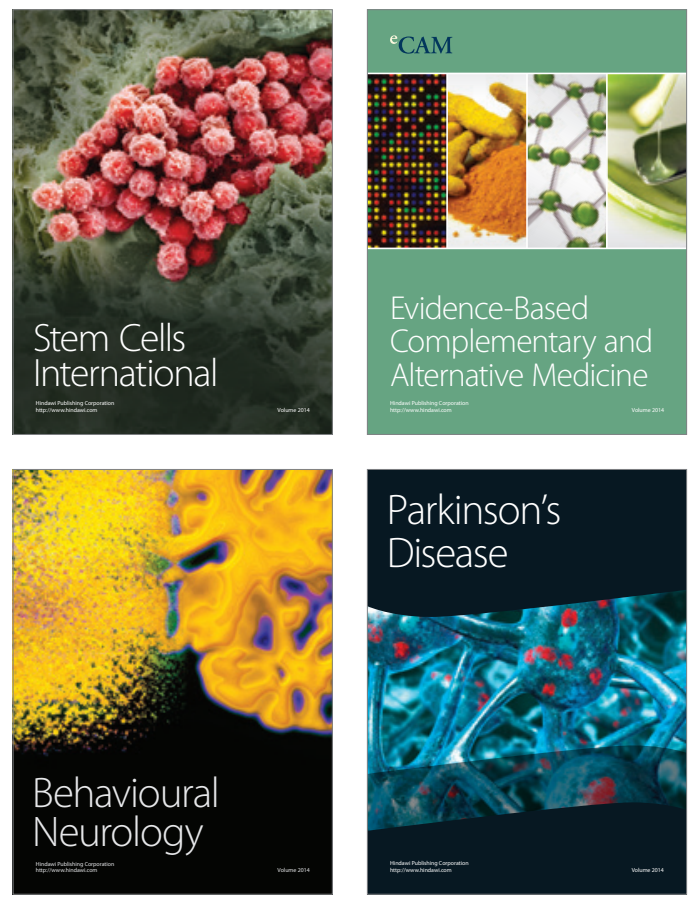
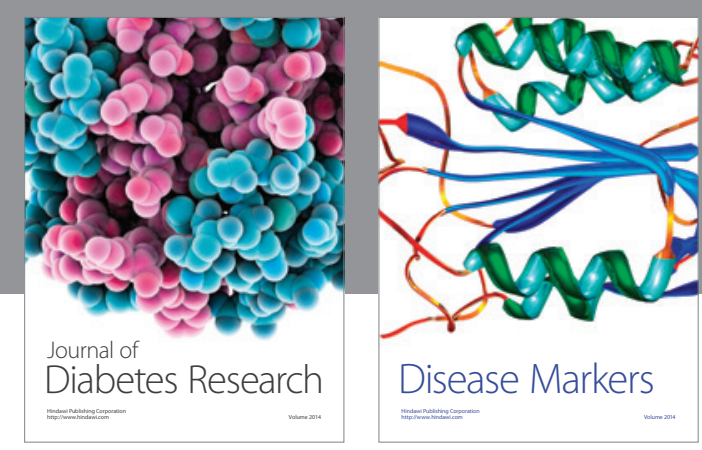

Disease Markers
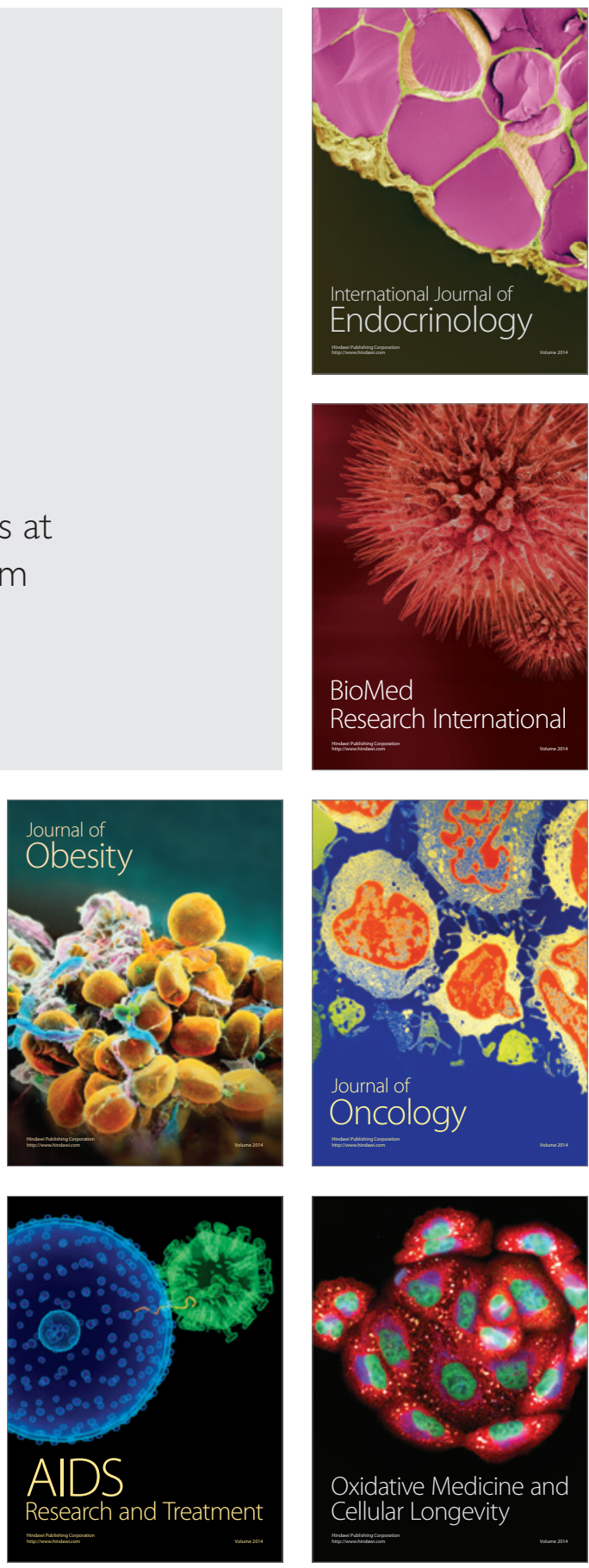